\title{
The Effect of Time-to-Surgery on Outcome in Patients with Neurological Deficits Caused by Spinal Tuberculosis
}

\author{
Peng LIU, Hai NIE \\ Sichuan Academy of Medical Science, Sichuan Provincial People's Hospital, East Branch, Department of Orthopedic Surgery, Long Quan \\ District, Chengdu, China
}

\section{ABSTRACT}

AIM: To compare and analyze the influence of the duration of neurological symptoms and degree of neurological deficits on the postoperative neurological recovery of patients with neurological deficits caused by spinal tuberculosis (TB).

MATERIAL and METHODS: We retrospectively reviewed the data of 47 adult patients with neurological deficits caused by spinal TB. They were divided into three and four groups according to the duration of neurological symptoms and degree of neurological deficits, respectively. Differences in the mean rank within groups were statistically evaluated, and logistic regression of the two factors was performed to evaluate how these factors influence the recovery of neurological function.

RESULTS: Non-parametric tests indicated a significant difference among improvement grades in the three groups based on the duration of neurological symptoms $(\mathrm{p}<0.05)$. No significant difference was found among improvement grades of the patients preoperatively in the four groups based on the grade of neurological deficits $(p>0.05)$. Logistic regression showed that the preoperative grade of neurological deficits significantly influenced the improvement grade of neurological deficits $(p<0.05)$, but the correlation was not close $\left(\mathrm{R}^{2}=0.28\right)$.

CONCLUSION: The duration of neurological symptoms, but not the grade of neurological involvement, is correlated with postoperative neurological recovery of patients with neurological deficits caused by spinal TB. However, reducing preoperative chemotherapy does not significantly yield improved outcomes; therefore, undergoing four weeks of preoperative chemotherapy is acceptable.

KEYWORDS: Spinal tuberculosis, Neurological deficit, Neurological symptom

\section{INTRODUCTION}

$\mathrm{T}$ Tuberculosis (TB) is a deadly disease affecting numerous individuals worldwide, particularly in developing countries. According to the World Health Organization (WHO), approximately three million individuals die annually because of this disease (1). The prevalence of TB has increased because of the human immunodeficiency virus (HIV) epidemic and drug resistance (7). This type of chronic wasting disease consumes considerable social and medical resources; as such, a heavy burden is placed on social and economic development.
Spinal TB is a specific orthopedic infection that affects the musculoskeletal system in 3 to $5 \%$ of HIV-negative patients and $60 \%$ of HIV-positive patients who are exposed to pulmonary infection (14). These statistical data emphasize the need to prepare for a future resurgence of spinal TB.

Paraplegia is among the most serious complications of spinal TB and is considered to be a difficult medical problem that requires surgery. Rapid and radical decompression may be useful for the recovery of neurological functions; however, surgery cannot possibly be performed under adequate chemotherapeutic cover until the general status of a patient 
is stabilized. Without the necessary intervention of anti-TB drugs, a single surgical treatment may cause hematogenous dissemination of TB that could endanger a patient's life. Hence, no unanimity on the best treatment form for this disease exists $(8,11,12)$. As such, surgeons contemplate the application of surgery to treat patients with neurological deficits caused by spinal TB. Two main factors should be considered for selection of a reasonable surgical opportunity: (1) affirmation of anti-TB therapeutic effect and (2) minimization of delays in the preoperative preparation of patients with neurological deficits caused by spinal TB; therefore, that surgery can be more conducive to neurological recovery. However, studies on this type of treatment are limited. In the present study, 47 adult patients with neurological deficits caused by spinal TB were observed to compare and analyze the relationship of the duration of neurological symptoms and the degree of neurological deficits with postoperative neurological recovery. We then proposed reasonable opportunities for surgery.

\section{MATERIAL and METHODS}

\section{General Data of Patients}

A total of 47 consecutive patients (24 males and 23 females) satisfied our inclusion/exclusion criteria. The following inclusion criteria were considered: (1) definitive spinal TB confirmed by postoperative pathology reports; (2) neurological deficits, including paraplegia or spinal cord compression; (3) age $\geq 18$ years; and (4) short-course chemotherapy administered in conjunction with the partial excision of pathologic vertebrae between June 2006 and May 2013 in a medical center. The following exclusion criteria were considered: (1) incomplete hospitalization data; (2) follow-up period of less than two years; and (3) known severe diabetes, cerebrovascular, and peripheral vascular diseases (to exclude interference of neurological symptoms). Patients' ages ranged from 22 years to 73 years with an average age of 45 years. Average weight was $52.3 \mathrm{~kg}$. Spinal TB was detected in thoracic $(n=25)$, lumbar $(n=11)$, and thoracolumbar $(n=11)$ spines. In the observed cases, 9 patients exhibited one pathologic vertebra, 21 patients displayed two pathologic vertebrae, 12 patients revealed three pathologic vertebrae, and 5 patients showed more than four pathologic vertebrae. The intervertebral destruction measured 2 to $8 \mathrm{~cm}$ with an average of $4.7 \mathrm{~cm}$.

\section{Neurological Involvement}

The duration of neurological symptoms ranged from 1 to
84 weeks with an average of 14 weeks. The durations of neurological symptoms such as paresthesia or myasthenia were shorter than 4 weeks $(n=10)$, between 4 and 8 weeks $(n=15)$, and longer than 8 weeks $(n=22)$. The patients preoperatively belonged to grades $A, B, C$, or $D(n=2,4,13$, or 28 , respectively) and postoperatively recovered to grades $A, B$, $C$, $D$, or $E(n=1,3,2,13$, or 28 , respectively; Table I) according to the American Spinal Injury Association (ASIA) Impairment Scale (3). All of the patients exhibited definite surgical indications, including vertebral body collapse or kyphosis, wide paravertebral abscesses, and sinuses unresponsive to medical treatment besides neurological deficits, including paraplegia or spinal cord compressions.

\section{Treatment and Surgical Methods}

All of the patients were subjected to preoperative imaging tests, such as X-ray, computed tomography (CT), or magnetic resonance imaging (MRI), which were consistent with their postoperative pathology reports. These patients also received a $2 \mathrm{HRZE} / 8 \mathrm{H}_{2} \mathrm{R}_{2} \mathrm{E}_{2}$ treatment based on the chemotherapeutic guidelines recommended by $\mathrm{WHO}$ National Programs of Treatment of Tuberculosis (13). In detail, the patients were treated with daily oral doses of Isoniazid 0.3 , Rifampicin 0.45 , Ethambutol 0.75, and Pyrazinainide 1.5 for at least two weeks (an average of 3.2 weeks). Surgery was only allowed when TB poisoning symptoms were! mitigated; surgery was also recommended when erythrocyte sedimentation rate (ESR) was $<60 \mathrm{~mm} / \mathrm{h}$ or presented a decreasing trend. All patients were subjected to debridement and spinal fusion with an anterior or posterior approach (Figures 1A-D; 2A-D). Different suraical techniques were emploved to install fixation devices and correct kyphosis deformity, reconstruct the vertebrae height and stabilize the spine. The TB focus was then directly resected by an ostectrite. Autogenous ribs or iliac bones were collected, cut into small pieces, and filled into a mesh of titanium as a vertebral body prosthesis. This prosthesis was placed into the space of the veltebra left by debridement, and an internal fixation plate was installed. The wound was washed again with frozen saline solution; pills containing streptomycin $(1.0 \mathrm{~g})$ and isoniazid $(0.3 \mathrm{~g})$ were placed, a closed thoracic drainage (if thoracic) or drainage (if lumbar) tube was used, and wounds were sutured. Anti-TE3 treatment was administered postoperatively. Informed corisents were provided by the patients before they were subjected to surgery. The approval of the institutional review boarcl was also obtained.

Table I: Preoperative Neurological Deficits, Postoperative Neurological Recovery, and Improvement Grade of Patients with Spinal Tuberculosis

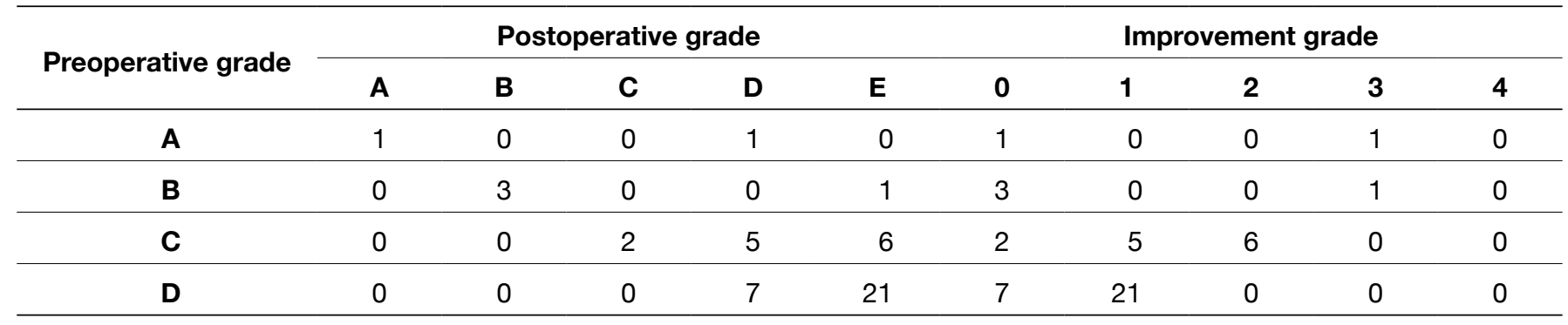




\section{Data Collection and Statistical Analyses}

The medical records of all of the patients were collected. The duration of preoperative neurological symptoms and the degree of neurological deficits were considered as grouping variables. The patients were divided into three groups based on the duration of neurological symptoms before surgery: shorter than four weeks, from four weeks to eight weeks and longer than eight weeks. The patients were further divided into four groups according to the preoperative ASIA Impairment Scale results: ASIA A, B, C and D. The postoperative improvement grade of neurological recovery of the patients was considered to be a test variable. Analyses were executed using SPSS version 13.0 (SPSS UK Ltd., Woking, United Kingdom). Differences in the mean rank within groups were statistically evaluated by non-parametric tests with a $\mathrm{K}$ independent sample test. Postoperative improvement grade of neurological function (with and without effect), duration of neurological symptoms and neurological deficit grade were subjected to logistic regression to establish a mathematical model of the effect of these factors on the recovery of neurological function. $p<0.05$ was considered significant. The protocol of this study was approved by the ethical committee of Sichuan Provincial People's Hospital.

\section{RESULTS}

\section{Clinical Outcomes}

With the exception of one patient, all patients were generally in good postoperative conditions, and systemic TB symptoms disappeared by three months postoperation. Clinical test results, such as ESR and C-reactive protein, were within normal or basically normal ranges (ESR $<15 \mathrm{~mm} / \mathrm{h}$, C-reactive protein $<10 \mathrm{mg} / \mathrm{L}$ ); there was one patient whose symptom was exacerbated because of irregular postoperative medication. Fusion occurred in all patients after a two to five year followup period with an average of 3.9 years. The average length of hospital stay was 13.5 days. Postoperative complications included pulmonary atelectasis or intercostal neuralgia
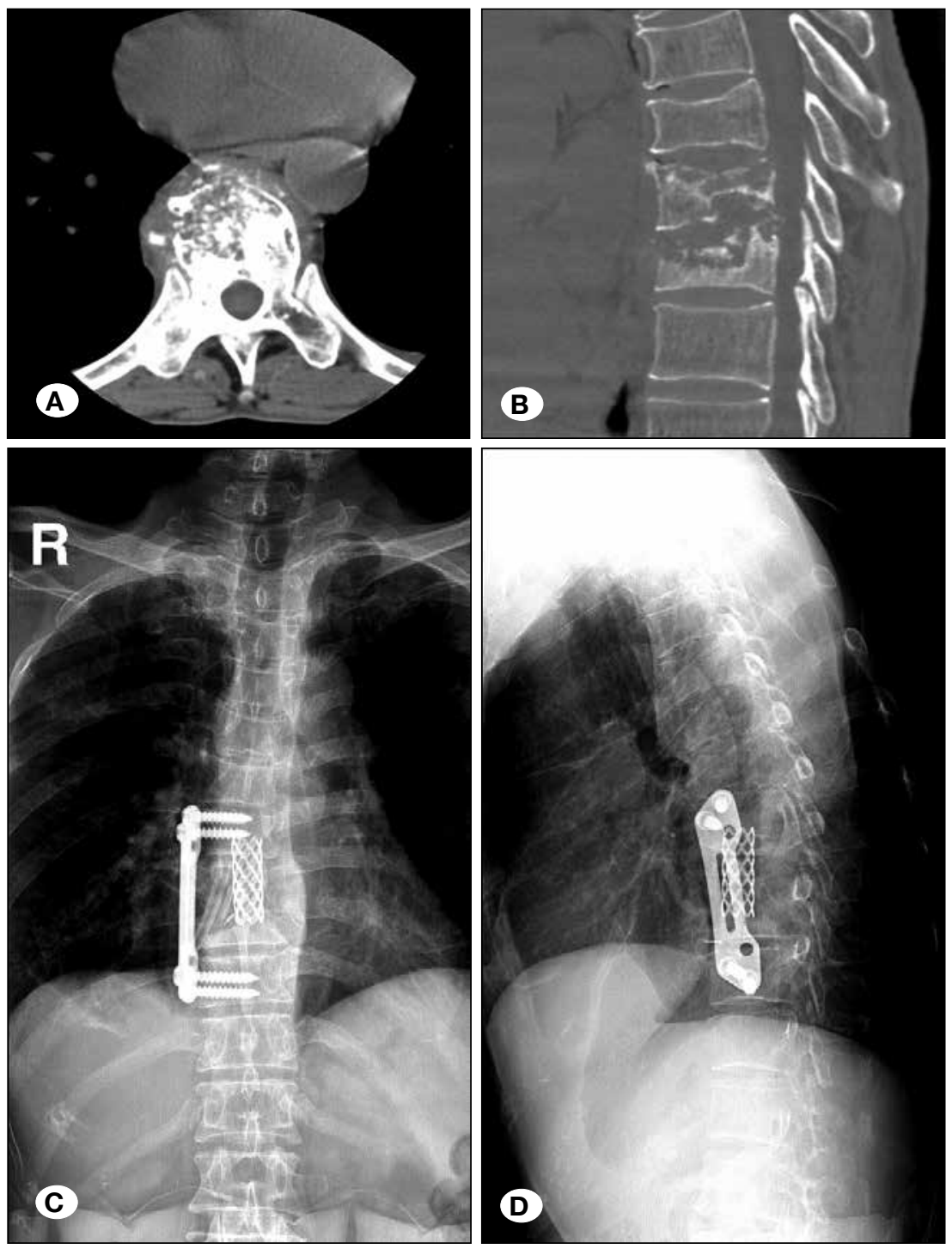

Figure 1: Axial (A) and sagittal (B) CT scans of a patient with spinal tuberculosis in T 8-9 who underwent debridement and spinal fusion with an anterior approach. Anteroposterior (C) and lateral (D) radiographs of a patient who had preoperative neurological deficits classified under grade $D$ and improved to grade $E$ within a three-month-postoperative follow-up according to the ASIA Impairment Scale. The symptoms of poisoning disappeared and the systemic condition improved. 
(accounted for 12\% in thoracic TB) and abdominal distention or meralgia paresthetica (accounted for $18 \%$ in lumbar TB). These complications were appropriately treated and disappeared after one week.

\section{Statistical Results}

The average improvement grades of the patients whose durations of neurological symptoms were less than four weeks, four weeks to eight weeks, and more than eight weeks were $0.50,0.87$, and 0.73 , respectively. The average improvement grades of the patients with neurological deficits classified as classes $A$ to $D$ were $0.50,0.25,0.83$, and 0.75 , respectively. Differences in the mean rank within groups were statistically evaluated. A significant difference was found among the improvement grades in the three groups based on the duration of neurological symptoms according to the ASIA Impairment Scale $(p<0.05)$. The mean rank (Table II) of the group with a four to eight week neurological symptom duration was 29.77 , which was higher than that in the group with less than four weeks (16.75) and longer than that with eight weeks of neurological symptom (23.36). No significant difference was found among the improvement grades of the patients in the four groups based on the grade of neurological deficits according to the ASIA Impairment Scale ( $p>0.05$; Table III). The two factors were subsequently analyzed by logistic regression. The results indicated the level of neurological impairment significantly influenced the improvement grade of neurological deficits $(p<0.05)$, but the correlation was not close $\left(R^{2}=0.28\right)$.

\section{DISCUSSION}

\section{Surgical Treatment of TB}

Surgery is considered to be an auxiliary treatment of spinal TB because most osteoarticular TB cases can be cured by anti-TB drugs $(10,16,17)$. Surgical treatments combined with chemotherapy exhibits several advantages, such as signifi-
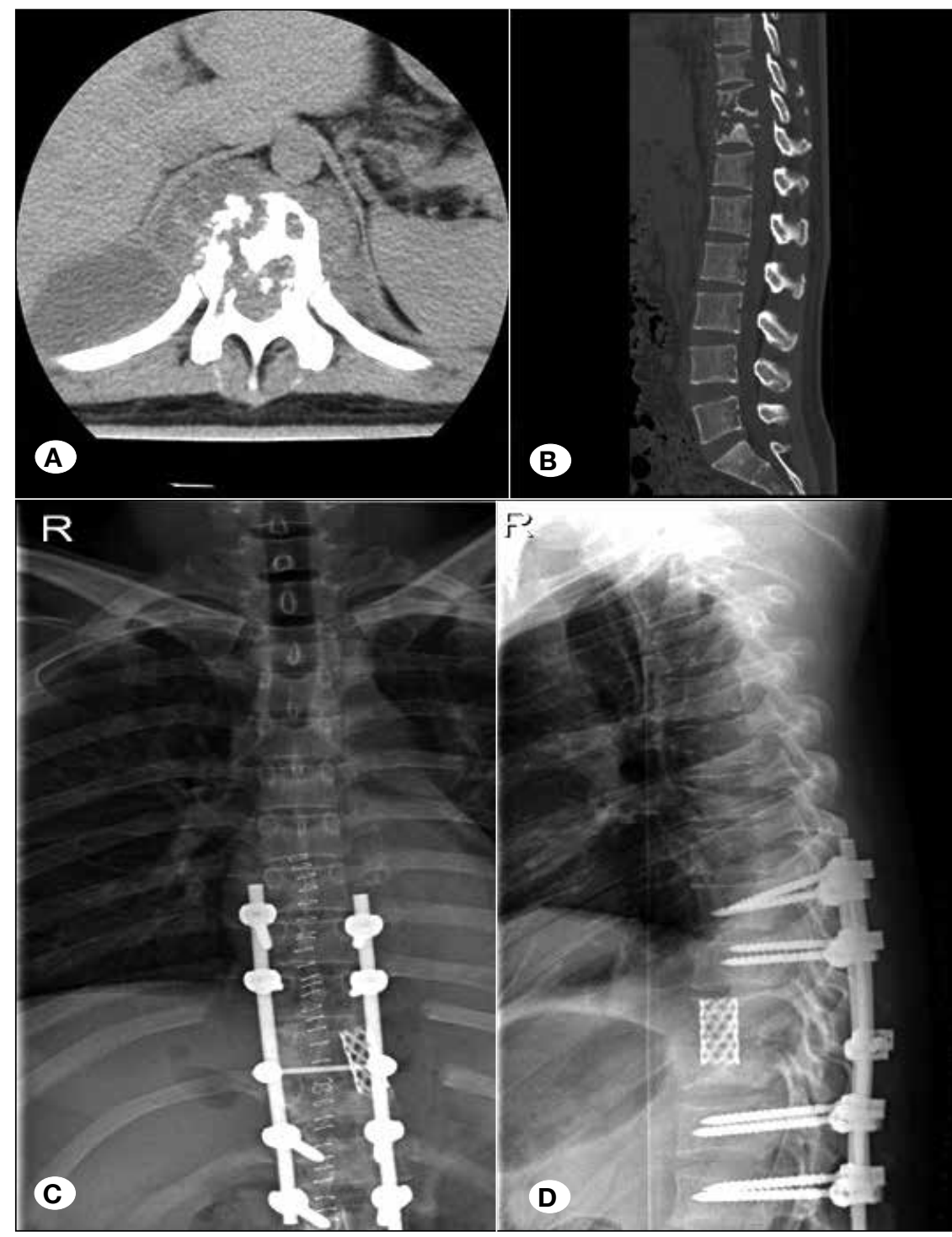

Figure 2: Axial (A) and sagittal (B) CT scans of a patient with paraplegia caused by spinal tuberculosis in T 9-11 who underwent debridement and spinal fusion with a posterior approach. Anteroposterior (C) and lateral (D) radiographs of a patient who had preoperative neurological deficits classified under grade $B$ and improved to grade $E$ within a three-monthpostoperative follow-up according to the ASIA Impairment Scale. The neurological deficits evidently improved. 
Table II: Improvement Grade of Patients with Neurological Deficits Caused by Spinal Tuberculosis according to the ASIA Impairment Scale in the Three Groups Based on Symptom Duration

\begin{tabular}{|c|c|c|c|c|}
\hline \multirow{2}{*}{$\begin{array}{l}\text { Improvement } \\
\text { grade }\end{array}$} & \multicolumn{3}{|c|}{ Duration of neurological symptoms (from onset of symptoms to time of surgery) } & \multirow{2}{*}{ Tota } \\
\hline & $<4$ weeks & 4-8 weeks* & $>8$ weeks & \\
\hline 0 & 5 & 2 & 6 & 13 \\
\hline 1 & 5 & 8 & 13 & 26 \\
\hline 2 & 0 & 3 & 3 & 6 \\
\hline 3 & 0 & 2 & 0 & 2 \\
\hline
\end{tabular}

*indicate significant difference to the other two groups at $p<0.05$.

Table III: Improvement Grade of Patients with Neurological Deficits Caused by Spinal Tuberculosis according to the ASIA Impairment Scale in Four Groups Based on the Grade of Neurological Deficits

\begin{tabular}{|c|c|c|c|c|c|}
\hline \multirow{2}{*}{$\begin{array}{l}\text { Improvement } \\
\text { grade }\end{array}$} & \multicolumn{4}{|c|}{ Grade of neurological deficits } & \multirow{2}{*}{ Total } \\
\hline & A & B & C & D & \\
\hline 0 & 1 & 3 & 2 & 7 & 13 \\
\hline 1 & 0 & 0 & 5 & 21 & 26 \\
\hline 2 & 0 & 0 & 6 & 0 & 6 \\
\hline 3 & 1 & 1 & 0 & 0 & 2 \\
\hline
\end{tabular}

No significant difference among the four groups at $p>0.05$.

cantly shortening the course and reducing the incidence of complications $(6,15,18)$. The involvement of neurological functions results in the most serious consequence. Thus, safe and effective neurological recovery is an important therapeutic intervention target for surgery. Surgery as treatment for Pott's paraplegia should be considered to provide the highest therapeutic effect to treat neurological deficits and simultaneously ensure the safety of surgical procedures.

Scholars believed that operative treatment for early neurological damage caused by abscess compression slightly affects patients with paraplegia caused by spinal TB; however, even conservative treatments that can successfully cure paraplegia require long-term bed rest. Surgery can rapidly improve neurological function recovery. The curative effect of surgery is also reliable; as such, surgery for these patients should be a primary treatment option (14). Any surgery requires tedious and essential preoperative preparation, particularly for patients with spinal TB, who are non-ambulant or have received prolonged outpatient treatment and for a majority of patients in poor mental and physical conditions because TB is a type of chronic wasting disease. Therefore, the function of chemotherapy as a basis to treat spinal TB is emphasized. A previous study suggested that the minimum chemotherapy time of patients with spinal TB before they undergo surgery should not be less than two weeks (11). However, a few scholars believed that the early decompression and stabilization of the spinal cord contribute to neurological recovery $(5,9)$. Thus, a therapeutic contradiction is possibly inevitable.

\section{The Analyses of Time-to-Surgery}

In our study, a total of 47 patients with neurological deficits caused by spinal TB were selected. The patients were divided into groups of three and four groups according to the duration of neurological symptoms and the grade of neurological damage, respectively. Differences in the mean rank within groups were statistically evaluated, and the two factors were subjected to logistic regression to evaluate the effect of these factors on the recovery of neurological function. Different statistical methods seem caused different results, but the final conclusion actually was not contradictory. The result of first statistical method ( $\mathrm{K}$ independent sample test) indicated that a significant difference was found among improvement grades in the three groups based on the duration of neurological symptoms. On the contrary, the result of second statistical method (logistic regression) indicated the grade of neurological involvement influenced the improvement grade of neurological function. But preoperative grade of neurological involvement was not closely correlated with postoperative neurological recovery. Because the Nagelkerke $R^{2}$ value was only 0.28 , which was far $<0.7$ (regarded as a close value); this correlation was not close and was negligible. Hence, we were inclined to accept the result of the first statistical method. After further analysis was conducted, the postoperative grade of neurological recovery in patients with neurological symptoms that lasted four to eight weeks was the highest among this group. Therefore, the duration of preoperative neurological symptoms, but not the level of neurological deficits, influenced the degree of recovery; however, a short duration 
did not indicate enhanced effects. For this phenomenon, the pathological change in spinal TB possibly started as a result of chronic vertebral destruction and kyphotic deformity that subsequently induced the compression of the spinal cord, thereby causing neurological deficits, which differ from an acute spinal cord injury. In other words, adaptations may be observed with chronic deformity, although the alleviation of all pressure changes in the vertebral canal by chronicity cannot be assumed (4). Hence, the therapeutic effect of rapid spinal cord decompression was not necessarily better than that of a slightly delayed decompression.

\section{Innovation and Deficiency of This Study}

Of course the rehabilitation of spinal TB is a complicated process that is influenced by many treatment factors such as surgical debridement, stabilization and appropriate chemotherapy. However, in this study, we observed how the two factors of disease itself (the duration of neurological symptoms and the grade of neurological involvement) influenced the rehabilitation of spinal TB and aimed to disclose the relationship between the time of preoperative chemotherapy and the degree of neurological recovery. Although all patients received different durations of preoperative chemotherapy (some of them received two or four weeks of chemotherapy or even longer), the comparison was based from the time of symptom onset to the time of surgery. The result of our study indicated that the mean rank of the group with four to eight weeks of neurological symptom duration was higher than that in the group with less than four weeks or longer than eight weeks of neurological symptoms. We deduced that no difference was present between preoperative chemotherapy administered for less than four weeks or more than four weeks because the preoperative chemotherapy time was not longer than the time of symptom onset to the time of surgery. An interval of four weeks was then selected as a criterion for grouping because of several factors: (1) preoperative chemotherapy should be guaranteed for at least two weeks for TB patients with neurological deficits and four weeks for TB patients without neurological deficits to suppress and control mycobacterium activity in vivo (2), and (2) baseline balancing was considered because patients with short-duration neurological symptoms are relatively rare. This phenomenon can be attributed to their late presentation in our center; forty percent (19 out of 47 ) of the patients already suffered from paraplegia when hospitalized. This finding is consistent with that observed in Asian patients who do not prefer to consult with a specialist for initial care (19). In the present study, all surgical procedures were administered by four senior spinal surgeons at one medical center so it was difficult to avoid some bias. The limited number of cases may cause baseline inequality in the groupings because patients with spinal TB belonging to grades $A$ and $B$ of the ASIA Impairment Scale ( $n=2$ and 4, respectively) were very rare. Pott's disease is a chronic illness; as such, patients often delay treatment. Thus, patients with a short-duration of neurological symptoms are relatively rare ( $n=10$, symptom duration $<4$ weeks).

\section{CONCLUSION}

Patients with neurological deficits caused by spinal TB should be actively treated surgically, regardless of the difference in the duration of neurological symptoms or grade of neurological damage. As long as proper surgical treatments are applied, a patient may recover partial neurological function. Furthermore, the duration of neurological symptoms, but not the grade of neurological involvement, is correlated with postoperative improvement of neurological function of patients with neurological deficits caused by spinal TB. However, reducing preoperative chemotherapy time does not significantly yield improved outcomes; undergoing four weeks of preoperative chemotherapy is still acceptable for patients with neurological deficits. Hence, surgery is beneficial for patients with neurological deficits, particularly paraplegia caused by spinal TB, even if neurological symptoms have been present for a substantial period of time.

\section{REFERENCES}

1. Adams JC: Technique, dangers and safeguards in osteotomy of the spine. J Bone Joint Surg Br 34: 226-232, 1952

2. Alici E, Akcali O, Tatari H, Gunal I: Effect of preoperative chemotherapy on the outcome of surgical treatment of vertebral tuberculosis: Retrospective analysis of 434 cases. Arch Orthop Trauma Surg 121:65-66, 2001

3. ASIA International Standards for Neurological Classification of Spinal Cord Injury, Revised 2002. Chicago, IL: American Spinal Injury Association, 2002

4. Farley CW, Curt BA, Pettigrew DB, Holtz JR, Dollin N, Kuntz C 4th: Spinal cord intramedullary pressure in thoracic kyphotic deformity: A cadaveric study. Spine (Phila Pa 1976) 37:E224230,2012

5. Fehlings MG, Perrin RG: The role and timing of early decompression for cervical spinal cord injury: Update with a review of recent clinical evidence. Injury 36: 13-26, 2005

6. Ghadouane M, Elmansari O, Bousalmame N, Lezrek K, Aouam $\mathrm{H}$, Moulay I: Role of surgery in the treatment of Pott's disease in adults. Apropos of 29 cases. Rev Chir Orthop Reparatrice Appar Mot 82:620-628,1996 (In French)

7. Griffiths LI, Herbert S, Ball J, Wallace F, Kemp HB, Konstam PG: A five-year assessment of controlled trials of in-patient and out-patient treatment and of plaster-of-Paris jackets for tuberculosis of the spine in children on standard chemotherapy. Studies in Masan and Pusan, Korea. Fifth report of the Medical Research Council Working Party on tuberculosis of the spine. J Bone Joint Surg Br 58:399-411, 1976

8. Guven O, Kumano K, Yalcin S, Karahan M, Tsuji S: A single stage posterior approach and rigid fixation for preventing kyphosis in the treatment of spinal tuberculosis. Spine (Phila Pa 1976) 19:1039-1043, 1994

9. HabibAG:Aclinical and epidemiologic update on the interaction between tuberculosis and human immunodeficiency virus infection in adults. Ann Afr Med 8:147-155, 2009

10. Herbert S, Wallace F, Konstam PG, Scadding JG, Stott $\mathrm{H}$, Sutherland I: A controlled trial of ambulant out-patient treatment and in-patient rest in bed in the management of tuberculosis of the spine in young Korean patients on standard chemotherapy a study in Masan, Korea. First report of the Medical Research Council Working Party on Tuberculosis of the Spine. J Bone Joint Surg Br 55:678-697, 1973 
11. Krengel WF 3rd, Anderson PA, Henley MB: Early stabilization and decompression for incomplete paraplegia due to a thoracic-level spinal cord injury. Spine (Phila Pa 1976) 18:2080-2087, 1993

12. Kumar K: The penetration of drugs into the lesions of spinal tuberculosis. Int Orthop 16:67-68, 1992

13. Liu P, Sun M, Li S, Wang Z, Ding G: A retrospective controlled study of three different operative approaches for the treatment of thoracic and lumbar spinal tuberculosis: Three years of follow-up. Clin Neurol Neurosurg 128:25-34, 2015

14. Moon MS: Tuberculosis of the spine. Controversies and a new challenge. Spine (Phila Pa 1976) 22:1791-1797, 1997

15. Parthasarathy R, Sriram K, Santha T, Prabhakar R, Somasundaram PR, Sivasubramanian S: Short-course chemotherapy for tuberculosis of the spine. A comparison between ambulant treatment and radical surgery-ten-year report. J Bone Joint Surg $\mathrm{Br}$ 81:464-471, 1999
16. Pattisson PR: Pott's paraplegia: An account of the treatment of 89 consecutive patients. Paraplegia 24:77-91, 1986

17. Tuli SM, Kumar K, Sen PC: Penetration of antitubercular drugs in clinical osteoarticular tubercular lesions. Acta Orthop Scand 48:362-368, 1977

18. Wang Z, Ge Z, Jin W, Qiao Y, Ding H, Zhao H, Lin Z, Chen J, Yang, W: Treatment of spinal tuberculosis with ultrashortcourse chemotherapy in conjunction with partial excision of pathologic vertebrae. Spine J 7:671-681, 2007

19. Wong MD, Asch SM, Andersen RM, Hays RD, Shapiro MF: Racial and ethnic differences in patients' preferences for initial care by specialists. Am J Med 116:613-620, 2004 